\title{
Rare presentation of metastatic adenocarcinoma of prostate as inguinal lymphadenopathy and its management
}

\author{
Ajay G. Nimbalkar ${ }^{1 *}$, Sony B. Mehta², Vinal T. More ${ }^{1}$ \\ ${ }^{1}$ Department of General Surgery, Mumbai Port Trust Hospital, Mumbai, India \\ ${ }^{2}$ Department of Urology, Mumbai Port Trust Hospital, Mumbai, India \\ Received: 14 November 2020 \\ Accepted: 30 December 2020 \\ *Correspondence: \\ Dr. Ajay G. Nimbalkar, \\ E-mail: ajaynimbalkar0102@gmail.com \\ Copyright: (C) the author(s), publisher and licensee Medip Academy. This is an open-access article distributed under \\ the terms of the Creative Commons Attribution Non-Commercial License, which permits unrestricted non-commercial \\ use, distribution, and reproduction in any medium, provided the original work is properly cited.
}

\begin{abstract}
Prostate cancer is among the top ten cancers in India. Approximately half of patients with prostate cancer have metastasis on presentation, bones and regional lymph nodes being most common. A case of metastasised cancer of prostate presenting only with an inguinal mass without any other symptom is rare. A 72-year-old presented with a right inguinal mass for six months without any other complaints. Biopsy of the inguinal mass was done, and histopathology showed metastatic adenocarcinoma. His serum PSA (prostate specific antigen) was $117.325 \mathrm{ng} / \mathrm{ml}$. PET CT (positron emission tomography-computed tomography) showed PSMA (prostate specific membrane antigen) expressing lesion in left lobe of prostate involving left seminal with metastatic pelvic, right inguinal, retroperitoneal and mediastinal adenopathy with increased tracer uptake in sclerotic skeletal lesions in right iliac bone, acetabulum and right femoral shaft. TRUS (trans rectal ultra-sonography) guided biopsy of prostate (12 CORE) was performed and showed conventional prostatic adenocarcinoma with maximum Gleason score $4+4=8$, grade group IV. The patient's clinical stage was T3b N1 Mb. Patient is currently on abiraterone with degarelix with normal repeat serum PSA $(0.067 \mathrm{ng} / \mathrm{ml})$ on follow up at 5 months. Metastatic prostatic carcinoma usually presents with iliac nodes or bony metastasis, but rarely they present with inguinal lymphadenopathy and should be dealt with high clinical suspicion as they are already in advanced stage of the disease process.
\end{abstract}

Keywords: Prostate, Inguinal, Cancer, Adenocarcinoma, Metastasis

\section{INTRODUCTION}

Prostate is among the top ten cancers in India with 25,696 new cases, 17,184 deaths and 47,558 cases in 5-year prevalence. ${ }^{1}$ Approximately one-half of patients with prostate cancer have metastatic lesions at presentation. Bones and regional lymph nodes are the most common sites of metastasis. Prostate cancer spreads via the lymphatics from the obturator lymph nodes to the presacral, lateral sacral, internal and external iliac, and inguinal lymph nodes. Inguinal lymphadenopathy is also a manifestation of carcinoma, metastatic disease, lymphoma, and leukaemia. In such cases, biopsy of the lymphadenopathy can be an effective diagnostic tool. ${ }^{2}$ But a patient presenting only with inguinal mass without any other symptoms is rare and requires high level of clinical suspicion to detect these cases early.

\section{CASE REPORT}

A 72-year-old male a known case of hypertension presented with a right inguinal swelling for 6 months. He had no history of any other complaint and a trivial trauma brought his attention to the swelling. Clinical examination revealed a single, hard, fixed right inguinal horizontal group lymph node of $4 \times 4 \mathrm{~cm}$. Abdomen was normal and on digital rectal examination there was grade 2 prostatomegaly with hard left lobe. Biopsy of the inguinal mass was done, and histopathology showed metastatic adenocarcinoma. His serum PSA was 117.325 
$\mathrm{ng} / \mathrm{ml}$. Rest of the tumor markers were normal. Contrastenhanced CT showed a bulky and heterogeneously enhancing left hemi prostate and left seminal vesicle with periprostatic fat stranding and multiple significant mediastinal, retroperitoneal, common and external iliac and right inguinal lymph nodes. PET CT showed PSMA expressing lesion in left lobe of prostate involving left seminal with metastatic pelvic, right inguinal, retroperitoneal and mediastinal adenopathy with increased tracer uptake in sclerotic skeletal lesions in right iliac bone, acetabulum and right femoral shaft. TRUS guided biopsy of prostate (12 CORE) was performed and showed conventional prostatic adenocarcinoma with maximum Gleason score $4+4=8$, Grade group IV. The patient's clinical stage was T3b N1 $\mathrm{Mb}$. Patient and relative were explained about disease status and all treatment options. Patient chose medical castration and Abiraterone as treatment. Patient is currently on abiraterone with degarelix with normal repeat serum PSA $(0.067 \mathrm{ng} / \mathrm{ml})$ on follow up at 5 months.

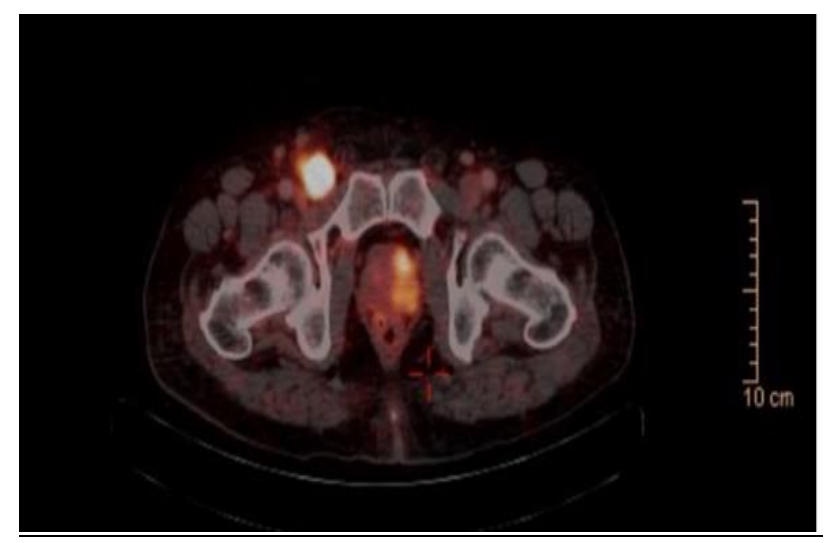

Figure 1: PET CT of increased tracer uptake in left prostate and right inguinal lymph node.

\section{DISCUSSION}

Prostate cancer usually spreads by direct, lymphatic and haematogenous dissemination. It spreads to the prostatic capsule, bladder base and seminal vesicles by direct invasion. Lymphatic spread usually occurs to the external and internal iliac, obturator, presacral and hypogastric nodes, and then to para-aortic lymph nodes. Prostate cancer mainly spreads through the lateral route, to the obturator nodes (medial chain of external iliac), then to the middle and lateral chains of the external iliac nodes. The other route is the internal iliac (hypogastric) route. Also, drainage occurs along an anterior route, via nodes located anterior to the urinary bladder and a presacral route anterior to the sacrum and the coccyx. Lymph node spread tends to be ipsilateral in patients with a primary tumour affecting only one lobe of prostate. Haematogenous spread occurs commonly to bone, lungs and liver. Many uncommon routes of lymphatic drainage have been reported, such as the periprostatic and seminal vesicle nodes, gonadal vessels, mesenteric and mesocolic nodes, posterior iliac crest nodes and inferior phrenic nodes. ${ }^{3}$ It is also possible that malignant cells can reach the inguinal nodes via spermatic cord through localised spread or from ectopic prostate tissue outside the genitourinary system. ${ }^{4}$ Inguinal lymphadenopathy can also occur due to metastasis from the urethral, penile and anal canal, and from lower limb cancers, lymphoma, leukaemia, infections and sexually transmitted diseases. Excision biopsy is ideal because the larger sample will allow for better chances of detecting cancer cells if they are present. ${ }^{2}$ Hence, adenocarcinoma of the prostate should be considered as an important cause for inguinal lymphadenopathy and this stresses the importance of clinical examination.

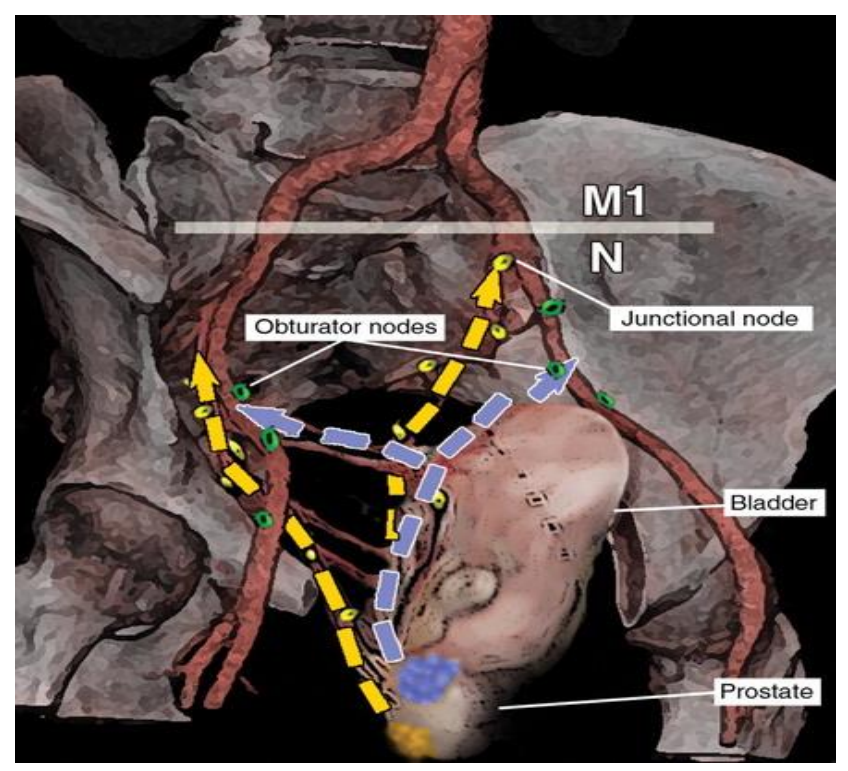

Figure 2: Common pathways of metastasis from prostate cancer: the obturator nodes in the external iliac (green) nodal group and the internal iliac (yellow) nodes in hypogastric route (dashed yellow arrows). 5

\section{CONCLUSION}

Presentation of prostatic carcinoma with inguinal mass is rare as they usually present with iliac nodes or bony metastasis. Patients presenting with inguinal lymphadenopathy should be dealt with high clinical suspicion as they are already in advanced stages of the disease process.

\section{Funding: No funding sources Conflict of interest: None declared Ethical approval: Not required}

\section{REFERENCES}

1. Ferlay J, Soerjomataram I, Dikshit R, Eser S, Mathers $\mathrm{C}$ et al. Cancer incidence and mortality worldwide: sources, methods and major patterns in globocan 2012. Int J Cancer. 2015;136(5):E359-86. 
2. Komeya M, Sahoda T, Sugiura S, Sawada T, Kitami $\mathrm{K}$. A case of metastatic prostate adenocarcinoma to an inguinal lymph node. Cent European J Urol. 2012;65(2):96-7.

3. Doreswamy K, Karthikeyan VS, Nagabhushana M, Shankaranand B. Prostatic adenocarcinoma presenting as isolated inguinal lymphadenopathy. BMJ Case Rep. 2015;2015:bcr2015210825.

4. Lin H, Chang T, Li C, Huang C. Metastatic Prostate Cancer Found Incidentally during an Inguinal Herniorrhaphy: A Case Report. J Taiwan Urological Asso. 2007;18(1):39-41.
5. Paño B, Sebastià C, Buñesch L, Mestres J, Salvador R, Macías NG et al. Pathways of lymphatic spread in male urogenital pelvic malignancies. Radiographics. 2011;31(1):135-60.

Cite this article as: Nimbalkar AG, Mehta SB, More VT. Rare presentation of metastatic adenocarcinoma of prostate as inguinal lymphadenopathy and its management. Int Surg J 2021;8:746-8. 